\title{
Role of Postoperative Antibiotics for prevention of infection after appendectomy in patients with complicated appendicitis
}

Poudel R ${ }^{1}$

Department of Surgery, Universal College of Medical Sciences, Bhairawha

Correspondence: Dr R. Poudel, Assistant Professor, Department of Surgery, Universal College of Medical Sciences Email: rajeshpoudel@yahoo.com

\begin{abstract}
Background: Acute Appendicitis is the most common general surgical emergency. Despite improved asepsis and surgical techniques, postoperative complications are still common. The general assumption is that it is better to treat patients with complicated appendicitis (perforated or gangrenous) with antibiotics, in contrast to patients with simple appendicitis. Aim of this study was to compare outcomes of patients treated with antibiotics after appendectomy in complicated appendicitis with those of patients who were not treated with antibiotics.
\end{abstract}

Methods: This was a retrospective study carried out in Universal College of Medical Sciences, Bhairawha, Nepal. Patients who underwent emergency appendectomy in 2 years period were included in the study. Patients with complicated appendicitis were analyzed on the basis of whether they received postoperative antibiotics or not. Main outcome measured were wound complications and length of hospital stay.

Results: During the 2-year study period, a total of 203 patients met inclusion criteria. Complicated appendicitis was identified in 71 patients (34.97\%). Postoperative antibiotics were administered to 42 (59.2\%). On Univariate and multivariate analyses Postoperative antibiotics were not associated with decrease in wound infection. But it is associated with increase in length of hospital stay ( $p<0.01,95 \%$ confidence interval $2.68-6.05)$.

Conclusion: Use of Postoperative antibiotics does not decrease the rate of wound infection in patient with complicated appendicitis but result in increase length of hospital stay.

Keywords: Appendicitis, Complicated, Gangrenous, Perforated, Postoperative antibiotics 